Article

\title{
Subtilisin-Involved Morphology Engineering for Improved Antibiotic Production in Actinomycetes
}

\author{
Yuanting $\mathrm{Wu}^{1,2}$, Qianjin Kang ${ }^{1,2}$, Li-Li Zhang ${ }^{3}$ and Linquan Bai ${ }^{1,2,3, * \mathbb{C}}$ \\ 1 State Key Laboratory of Microbial Metabolism, School of Life Sciences \& Biotechnology, \\ Shanghai Jiao Tong University, Shanghai 200204, China; yuantingwu1987@sjtu.edu.cn (Y.W.); \\ qjkang@sjtu.edu.cn (Q.K.) \\ 2 Joint International Research Laboratory of Metabolic \& Developmental Sciences, \\ Shanghai Jiao Tong University, Shanghai 200240, China \\ 3 College of Life Science, Tarim University, Alar 843300, China; zhang63lyly@sina.com \\ * Correspondence: bailq@sjtu.edu.cn
}

Received: 3 May 2020; Accepted: 30 May 2020; Published: 3 June 2020

check for updates

\begin{abstract}
In the submerged cultivation of filamentous microbes, including actinomycetes, complex morphology is one of the critical process features for the production of secondary metabolites. Ansamitocin P-3 (AP-3), an antitumor agent, is a secondary metabolite produced by Actinosynnema pretiosum ATCC 31280. An excessive mycelial fragmentation of A. pretiosum ATCC 31280 was observed during the early stage of fermentation. Through comparative transcriptomic analysis, a subtilisin-like serine peptidase encoded gene $A P A S M \_4178$ was identified to be responsible for the mycelial fragmentation. Mutant WYT-5 with the APASM_4178 deletion showed increased biomass and improved AP-3 yield by $43.65 \%$. We also found that the expression of APASM_4178 is specifically regulated by an AdpA-like protein APASM_1021. Moreover, the mycelial fragmentation was alternatively alleviated by the overexpression of subtilisin inhibitor encoded genes, which also led to a $46.50 \pm 0.79 \%$ yield increase of AP-3. Furthermore, APASM_4178 was overexpressed in salinomycin-producing Streptomyces albus BK 3-25 and validamycin-producing S. hygroscopicus TL01, which resulted in not only dispersed mycelia in both strains, but also a $33.80 \%$ yield improvement of salinomycin to $24.07 \mathrm{~g} / \mathrm{L}$ and a $14.94 \%$ yield improvement of validamycin to $21.46 \mathrm{~g} / \mathrm{L}$. In conclusion, our work elucidates the involvement of a novel subtilisin-like serine peptidase in morphological differentiation, and modulation of its expression could be an effective strategy for morphology engineering and antibiotic yield improvement in actinomycetes.
\end{abstract}

Keywords: actinomycetes; transcriptome; morphology engineering; mycelial fragmentation; subtilisin

\section{Introduction}

The filamentous actinomycetes, with Streptomyces spp. as the major genus, are well known for their rich and diverse secondary metabolites, many of which have been developed into drugs (e.g., erythromycin, vancomycin) and agents for plant protection (e.g., avermectin, validamycin). On solid media, most of actinomycetes undergo a full cycle of morphological differentiation, initiated from spore germination, followed with the successive formation of vegetative mycelia, aerial mycelia and spore chains [1]. For industrial production of antibiotics in large scale, actinomycetes are usually subjected to submerged fermentation in liquid cultures. During submerged cultivation, the inoculated mycelia or mycelia germinated from spores start to form pellets, following which programmed cell death (PCD) occurs at the center, and new multinucleated mycelia develop inside or from the edge of the pellets to carry out antibiotic production [2]. In bioreactors, since the sizes and the densities of pellets are critical for nutrient and oxygen transfer, which are also triggering factors of PCD, mycelial morphology 
correlates with agitation, aeration, hydrodynamics and the yields of antibiotics [3]. Moreover, different antibiotic producing strains favor different morphologies. Whereas the pellet formation is required for nikkomycin production in Streptomyces tendae and erythromycin in Saccharopolyspora erythraea [4,5], it has negative effects on the production of tylosin in Streptomyces fradiae and nystatin in Streptomyces noursei [6,7]. Therefore, in order to optimize the production of antibiotics, morphology engineering of actinomycetes is needed. Classical strategies for morphology engineering, including adjustments of $\mathrm{pH}$, temperature, medium composition, aeration, and agitation, have been implemented for the production of lipstatin, $\varepsilon$-poly-L-lysine, rapamycin, etc. [8-10]. However, these manipulations usually affect large pellets and have limited effect on small pellets [11].

Morphology engineering by genetic manipulation is more targeted and flexible. Recently, several protein families have been identified to play roles in the control of morphogenesis [12]. The first family are proteins involved in the formation of tip-organizing center (TIPOC) for apical growth and hypha branching, including the DivIVA, cytoskeletal protein Scy, cell-wall remodeling protein SsgA, the cellulose synthase-like CslA, and its cognate galactose oxidase-like GlxA [13-17]. The second family of proteins involved in the control of liquid-culture morphogenesis include a cyclic nucleotide-binding protein EshA and amine oxidase HyaS [18,19]. The third family of proteins, such as poly- $\beta-1,6-N$-acetylglucosamine (PNAG) synthases MatAB, are responsible for extracellular polymer synthesis [20]. Overexpression of SsgA was employed to obtain fragmented mycelia and fast growth of Streptomyces venezuelae and Streptomyces coelicolor M145 in submerged cultures, which had positive effects on the productions of zincphyrin IV and undecylprodigiosin, respectively [21,22]. When gene hyaS was deleted in Streptomyces lividans, the production of undecylprodigiosin was substantially improved along with outgrowing bunches of hyphae [19]. Moreover, the deletion of genes mat $A$ and matB in S. lividans led to dispersed mycelia and improved production of tyrosinase [20].

The antitumor agent ansamitocins, structurally similar to maytansines from Maytenus serrata [23], are produced by several genera of actinomycetes, including Actinosynnema [24,25], Amycolatopsis [26,27] and Nocardiopsis [28]. For large scale fermentation, ansamitocins are produced with A. pretiosum subsp. auranticum ATCC 31565, and ansamitocin P-3 (AP-3) is the major product and has the most potent antitumor activity [24]. Using maytansinol as a payload, the deacylated product of AP-3, antibody-drug conjugates have been recently developed as new strategy for cancer treatment [29], e.g., the Food and Drug Administration (FDA)-approved T-DM1 (Kadcyla $\left.{ }^{\circledR}\right)$ for Human Epidermal Growth Factor Receptor 2 (HER2)-positive metastatic breast cancer [30]. Aided by the in-depth biosynthetic studies [31,32], the yield improvement of AP-3 has been intensively conducted through random mutagenesis and screening, process engineering, and pathway engineering, which includes the optimization of post-PKS modifications, enhancement of precursor supplies, improved gene expression, and so on [33-39]. However, the yield of AP-3 is still low and insufficient for supporting the clinical trials of drug leads in pipeline and subsequent clinical treatment.

Herein, an excessive mycelial fragmentation was found to be undesirable for the yield improvement of AP-3 in A. pretiosum subsp. pretiosum ATCC 31280. Through comparative transcriptomic analysis and gene inactivation, we identified a gene $A P A S M \_4178$, coding for a subtilisin-like serine peptidase, to be responsible for the mycelial fragmentation. The transcription of APASM_4178 was proven to be regulated by AdpA-like protein, and the mycelial fragmentation was alternatively alleviated by the overexpression of subtilisin inhibitor genes. Moreover, the overexpression of APASM_4178 led to dispersed mycelia and substantially improved yields of salinomycin and validamycin in corresponding producing strains.

\section{Materials and Methods}

\subsection{Strains, Plasmids, Media and DNA Techniques}

The bacterial strains, plasmids and primers used in this study are listed in Supplementary Tables $\mathrm{S} 1$ and S2. A. pretiosum ATCC 31280, the AP-3 producer, and its derivatives were cultured at $30{ }^{\circ} \mathrm{C}$ 
on YMG agar plates (0.4\% yeast extract, $1.0 \%$ malt extract, $0.4 \%$ glucose, $2.0 \%$ agar $(w / v)$, pH 7.2-7.3). For metabolites analysis, shake-flask fermentation was performed in 250-mL flask containing $30 \mathrm{~mL}$ of medium. The first seed medium (3.0\% tryptone soya broth powder, $0.5 \%$ yeast extract, and $5.0 \%$ sucrose $(w / v), \mathrm{pH} 7.5)$ was inoculated with agar-grown mycelia and cultivated at $30{ }^{\circ} \mathrm{C}, 220 \mathrm{rpm}$ for $24 \mathrm{~h}$. Subsequently, the second seed medium (3.0\% tryptone soya broth powder, $0.5 \%$ yeast extract, and $2.5 \%$ sucrose, $1.0 \%$ soluble starch $(w / v), 0.05 \%$ isobutanol and $0.05 \%$ isopropanol $(v / v), \mathrm{pH} 7.5)$ was inoculated with $1 \mathrm{~mL}$ of the first seed culture and incubated for $24 \mathrm{~h}$ at $30{ }^{\circ} \mathrm{C}, 220 \mathrm{rpm}$. Fermentation medium (yeast extract $0.8 \%$, malt extract $1.0 \%$, sucrose $1.5 \%$, soluble starch $2.5 \%(w / v)$, isobutanol $0.5 \%$, and isopropanol 1.2\% (v/v), $\mathrm{pH} 7.5)$ was inoculated with the second seed culture at $10 \%(v / v)$, and the fermentation continued for 8 days at $25{ }^{\circ} \mathrm{C}, 220 \mathrm{rpm}$. For the isolation of total DNA, A. pretiosum was cultivated in trypticase soy broth (TSB) [40] supplemented with $10.3 \%$ sucrose and $1 \%$ yeast extract at $30{ }^{\circ} \mathrm{C}$ for $24 \mathrm{~h}$.

\subsection{Growth Measurement and AP-3 Yield Determination}

The mycelial growth was estimated by determining the mycelial dry weight. Mycelia from $1.0 \mathrm{~mL}$ of fermentation broth were collected by centrifugation at 12,000 rpm for $2 \mathrm{~min}$, washed with $0.1 \mathrm{M}$ $\mathrm{HCl}$, and dried to constant weight at $65^{\circ} \mathrm{C}$ for dry cell weight (DCW) measurement.

To quantify AP-3 yield, the supernatant of the fermentation broth was extracted with two volumes of ethyl acetate and evaporated. The residue was dissolved in methanol, passed through $0.22-\mu \mathrm{m}$ filters, and subjected to high performance liquid chromatography (HPLC). HPLC analysis was operated on Agilent series 1260 (Agilent Technologies, Santa Clara, CA, USA) with an Agilent Eclipse Plus-C 18 column $(4.6 \times 150 \mathrm{~mm}, 5 \mu \mathrm{m})$, using a previously described method [39].

\subsection{Mycelial Morphology Observation}

Mycelial morphology was observed using phase-contrast microscopy and laser confocal microscopy. After being washed twice with an equal volume of phosphate-buffered saline (PBS), $10 \mu \mathrm{L}$ of mycelial suspension was pipetted onto a standard glass slide $(25 \times 75 \mathrm{~mm})$ and covered with cover slide $(24 \times 24 \mathrm{~mm})$. The images were captured with a digital camera CX41 mounted on the microscope (Olympus, Tokyo, Japan) with 400-fold magnification viewed under phase-contrast mode. For each sample, a minimum of five images were captured.

The dye FM 4-64FX (Molecular Probes, Inc., Eugene, OR, USA) with a concentration of $200 \mu \mathrm{M}$ in Hank's Balanced Salt Solution was used to dye the cell membrane. Equal volumes $(10 \mu \mathrm{L})$ of FM 4-64FX solution and mycelia suspension were mixed and kept on ice for $1 \mathrm{~min}$, and $10 \mu \mathrm{L}$ of the mixture was pipetted on a clean slide and covered by a cover slip. Samples were observed with an SP8 STED laser scanning confocal microscope (Leica Microsystems, Buffalo Grove, IL, USA), equipped with an oil-immersed 100× objective and a charge coupled device (CCD) camera. Samples were excited at a wavelength of $558 \mathrm{~nm}$ and observed at an emission wavelength of $734 \mathrm{~nm}$. Each mycelial sample was scanned at least for five times, and each scan was viewed in at least five fields.

\subsection{Transcriptome Sequencing and Analysis of A. pretiosum ATCC 31280}

The RNA for transcriptome sequencing was sampled at $15 \mathrm{~h}, 18 \mathrm{~h}$ and $24 \mathrm{~h}$ of fermentation. The total RNA was extracted using Trizol according to the manufacturer's instruction. The transcriptome sequencing was conducted at Shanghai Biotechnology Corporation (Shanghai, China), and the expression level of each gene was calculated as Fragments Per Kilobase of exon per Megabase of library size (FKPM). The transcriptomic data have been deposited at GEO (Gene expression Omnibus) with an accession number of GSE151010. With the collected data, the transcription levels between 15, 18, and $24 \mathrm{~h}$ were compared. Genes with the lowest transcription at $18 \mathrm{~h}$ were selected. 


\subsection{Transcription Analysis by Quantitative Real-Time PCR}

Mycelia in fermentation media were collected at different times. Total RNA was extracted with Redzol reagent according to the procedures described by the manufacturer (SBS Genetech, Beijing, China). The purity/concentration was assessed using Nanodrop 2000 (ThermoFisher, Waltham, MA, USA). Total DNA was removed by DNase I (ThermoFisher), and reverse transcription was carried out using RevertAid ${ }^{\mathrm{TM}}$ H Minus First Strand cDNA Synthesis Kit (ThermoFisher). The transcription of target genes was determined by quantitative real-time PCR (qRT-PCR) on a 7500 Fast Real-time PCR System (Applied Biosystems, Carlsbad, CA, USA) with One-Step PrimerScript ${ }^{\mathrm{TM}}$ RT-PCR Kit according to the manufacturer's procedure (Takara, Dalian, China). The transcription of target genes was internally normalized to the housekeeping gene $h r d B$ and quantified by the $2^{-\Delta \Delta C T}$ method [41]. Each reaction was performed in triplicate.

\subsection{Inactivation and Trans-Complementation of Gene APASM_4178}

To inactivate APASM_4178, the genomic DNA of A. pretiosum ATCC 31,280 (GenBank accession no. CP029607.1) was used as PCR template. A 1.38-kb XbaI-EcoRI fragment of the left flanking region and a 1.45-kb EcoRI-HindIII fragment of the right flanking region were ligated with XbaI/HindIII-digested plasmid pJTU1278 to generate plasmid pLQ855. The recombinant plasmid was transferred into Escherichia coli ET12567(pUZ8002) and then introduced into A. pretiosum ATCC 31,280 by intergeneric conjugation, and thiostrepton-resistant $\left(\right.$ Thio $^{\mathrm{R}}$ ) exconjugants were selected. Subsequently, APASM_4178-deletion mutant WYT-5 was selected from the initial Thio ${ }^{\mathrm{R}}$ exconjugants after two rounds of nonselective growth. Using primers $4178-\mathrm{ver}-\mathrm{F} / \mathrm{R}$, a $0.5-\mathrm{kb}$ fragment was amplified by PCR from the mutant WYT-5, whereas a 3.7-kb fragment was amplified from A. pretiosum ATCC 31280. Other genes were similarly inactivated.

For the trans-complementation of mutant WYT-5, gene APASM_4178 was amplified with primers 4178-F/R and cloned in NdeI/EcoRI-digested pLQ856 under the control of $k a s O p^{*}$ promoter. The recombinant plasmid pLQ874 was transferred into E. coli ET12567(pUZ8002) and then introduced into mutant WYT-5 by intergeneric conjugation. Apramycin-resistant $\left(\mathrm{Apr}^{\mathrm{R}}\right)$ exconjugants were selected and named as WYT-13.

\subsection{Overexpression of Subtilisin Inhibitor Genes}

For the overexpression of gene APASM_3064 in A. pretiosum ATCC 31280, APASM_3064 was amplified with primers 3064-F/R and cloned in NdeI/EcoRI-digested pLQ856 under the control of kasOp* promoter. The recombinant plasmid pLQ869 and control plasmid pLQ856 were individually transferred into E. coli ET12567(pUZ8002) and then integrated into the attB site of A. pretiosum ATCC 31,280 by intergeneric conjugation. Apramycin resistant $\left(\mathrm{Apr}^{\mathrm{R}}\right)$ exconjugants were selected. Derivative strain overexpressing gene APASM_6209 was similarly constructed.

\subsection{Heterologous Overexpression of the AdpA-Like Protein APASM_1021}

Gene APASM_1021 was amplified with primers 1021-F/R and cloned in HindIII/NdeI-digested pET28a. The recombinant plasmid pLQ864 was transformed into E. coli BL21(DE3) for protein overexpression. Colonies were picked and cultivated at $37^{\circ} \mathrm{C}$ in LB medium containing $50 \mu \mathrm{g} / \mathrm{mL}$ kanamycin until an optical density at $600 \mathrm{~nm}\left(\mathrm{OD}_{600}\right)$ of $0.6-0.8$ was reached. The culture was then induced with $0.1 \mathrm{mM}$ isopropyl- $\beta$-D-thiogalactoside (IPTG) and incubated further for $24 \mathrm{~h}$ at $16^{\circ} \mathrm{C}$. The induced cells were harvested, resuspended in $50 \mathrm{mM}$ Tris- $\mathrm{HCl}$ (pH 7.5), and disrupted by ultrasonication. For the purification of His-tagged APASM_1021, cells were harvested by centrifugation, resuspended with buffer A ( $20 \mathrm{mM}$ Tris- $\mathrm{HCl}, 300 \mathrm{mM} \mathrm{NaCl}, \mathrm{pH} 8.0)$ and sonicated for $30 \mathrm{~min}$ on ice, followed by centrifugation for $40 \mathrm{~min}$ at $12,000 \mathrm{rpm}$ and $4{ }^{\circ} \mathrm{C}$. Proteins from the supernatant were purified with Ni Sepharose $^{\mathrm{TM}} 6$ Fast Flow (GE Healthcare Life Sciences, Marlborough, MA, USA). 
The eluted His-tagged APASM_1021 was determined by 12\% sodium dodecyl sulfate-polyacrylamide gel electrophoresis (SDS-PAGE).

\subsection{Electrophoretic Mobility Shift Assays}

A carboxyfluorescein (FAM)-labeled DNA probe P4178 was amplified using primers 4178p-F-FAM/4178p-R (Table S2) and genomic DNA from A. pretiosum ATCC 31,280 as template. In a total volume of $20 \mu \mathrm{L}, 100 \mathrm{ng}$ FAM-labeled probes were incubated for $30 \mathrm{~min}$ at $30{ }^{\circ} \mathrm{C}$ with different amounts of purified His-tagged APASM_1021 in $1 \times$ binding buffer containing $10 \mathrm{mM}$ Tris-HCl (pH 7.5), $1 \mathrm{mM}$ EDTA, $100 \mathrm{mM} \mathrm{KCl}, 5 \%$ (v/v) glycerol, $0.1 \mathrm{DTT}$, and $0.01 \mathrm{mg} / \mathrm{mL}$ BSA [42]. The resulting DNA-protein complexes were then subjected to electrophoresis on $10 \%$ native polyacrylamide gels for $45 \mathrm{~min}$ at $4{ }^{\circ} \mathrm{C}$ [43]. The band shift was analyzed by XcitaBlue ${ }^{\mathrm{TM}}$ Conversion Screen Kit (Bio-Rad, Hercules, CA, USA).

\section{Results}

\subsection{An Early and Severe Mycelial Fragmentation in Liquid Cultures of A. pretiosum ATCC 31280}

In shake flask fermentation inoculated from a seed culture, the ansamitocin-producing A. pretiosum ATCC 31280 gained exponential growth in the first $24 \mathrm{~h}$, reached stationary phase at $48 \mathrm{~h}$, and maintained a relatively constant biomass $(14.95 \pm 0.64 \mathrm{mg} / \mathrm{mL}$ ) until day 6 (Figure 1A), albeit much lower than that of most actinomycetes. However, when we measured the biomass at three-hour intervals in the first two days, a sudden decrease was observed at $18 \mathrm{~h}$, followed by an increase from 21 to $24 \mathrm{~h}$. Interestingly, this decrease-then-increase pattern occurred again from 24 to $33 \mathrm{~h}$ (Figure 1B).

A

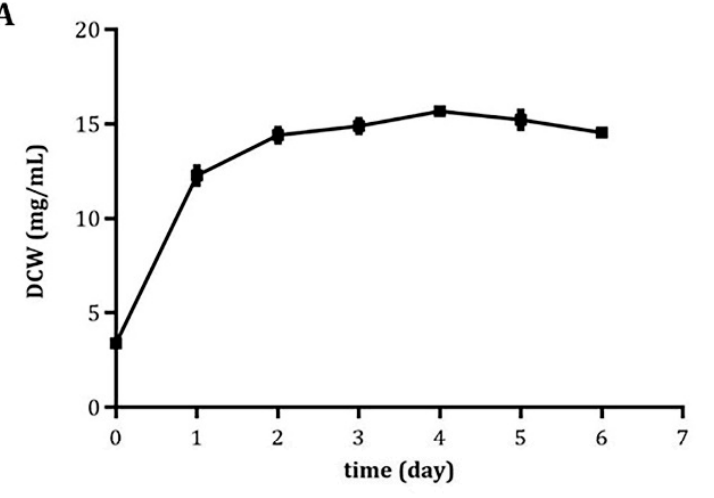

C

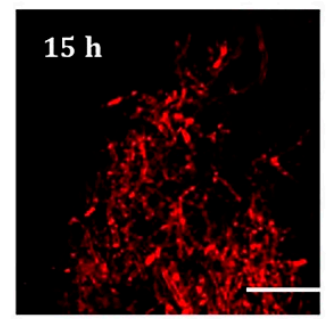

B

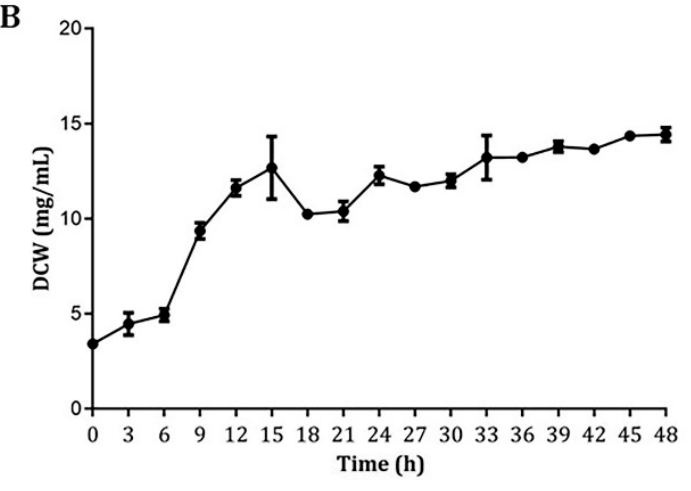

D

Figure 1. The growth and mycelial fragmentation of A. pretiosum ATCC 31280 in submerged cultures. (A) Growth curve of A. pretiosum ATCC 31280 during 6-day fermentation. Samples were taken daily. Error bars: mean \pm standard deviation (SD; $n=3$ biological replicates); (B) Growth of $A$. pretiosum ATCC 31280 during the first 48 -h fermentation. Samples were taken every $3 \mathrm{~h}$. Error bars: mean \pm SD ( $n=3$ biological replicates); (C) Confocal micrographs of A. pretiosum ATCC 31280 at 15, 18 or $24 \mathrm{~h}$. Scale bar: $10 \mu \mathrm{m}$. Magnification, 2500×; (D) Colony forming units (CFUs) of cultures at 15, 18 or $24 \mathrm{~h}$. 
For a better understanding of this phenomenon, mycelia of the fermentation broth were collected at 15,18 , and $24 \mathrm{~h}$ and checked under both phase-contrast light microscope (PCM) and confocal laser scanning microscope (CLSM). Stained with membrane-binding dye FM 4-64FX for CLMS, a severe fragmentation with short hyphae and less biomass was observed with the 18-h sample, whereas the mycelia of the 15 and 24-h samples formed thick clumps (Figure 1C). Moreover, the colony-forming units (CFUs) of each sample were quantified, and $2.0 \times 10^{7}, 1.0 \times 10^{7}$, and $32.5 \times 10^{7} \mathrm{CFUs} / \mathrm{mL}$ were found to be present in the 15, 18, and 24-h samples, respectively (Figure 1D), suggesting a concurrent cell lysis along with the mycelial fragmentation at $18 \mathrm{~h}$, which most likely led to the biomass decrease. In addition, five different media were used (Supplementary Table S3), and the mycelial fragmentation occurred in all of them around $18 \mathrm{~h}$ (data not shown), indicating that this unique fragmentation feature is genetically determined.

\subsection{Comparative Transcriptomic Analysis Identified a Subtilisin-Like Protease Gene Responsible for the Mycelial Fragmentation}

In order to identify genes involved in the early mycelial fragmentation, the total RNAs of mycelia collected at 15,18 , and $24 \mathrm{~h}$ were extracted and subjected to transcriptome sequencing using RNA-seq technology. We assumed that genes involved in the fragmentation have the lowest transcription at $18 \mathrm{~h}$ and therefore higher transcription at $15 \mathrm{~h}$ and $24 \mathrm{~h}$. According to this criterion, comparative analysis was subsequently performed among these three transcriptomes, and 12 genes were identified from a total of 7038 genes of the A. pretiosum ATCC 31,280 genome, with a $\geq 2$-fold higher transcription at $15 \mathrm{~h}$ and $24 \mathrm{~h}$ than that at $18 \mathrm{~h}$ (Supplementary Table S4). The transcription of these 12 genes was further verified through quantitative real-time PCR (qRT-PCR), among which 10 were found to keep the similar patterns with those obtained by transcriptome analysis. Genes APASM_1687 and APASM_4084 had continuously increased transcription from 15 to $24 \mathrm{~h}$ (Supplementary Figure S1).

These 12 candidate genes were individually deleted through double crossover homologous recombination to investigate any involvement in the fragmentation (Supplementary Figure S2). Whereas the deletions of 11 genes had no noticeable effect on the fragmentation (Supplementary Figure S3), the deletion of gene APASM_4178, coding for a subtilisin-family serine peptidase, led to a delayed fragmentation by $30 \mathrm{~h}$, from the original $18 \mathrm{~h}$ in the wild-type to $48 \mathrm{~h}$ in the mutant WYT-5 (Figure 2A). Moreover, the APASM_4178 deletion mutant WYT-5 had a $26.99 \%$ improved biomass of $22.77 \mathrm{mg} / \mathrm{mL}$ at day 4 and a $43.65 \%$ increased AP-3 yield (56.64 mg/L) compared with those of the wild-type (Figure 2B,C). Further confirmation came from the trans-complementation of the mutant WYT-5 with a cloned APASM_4178 under the control of $k a s O p^{*}$ promoter on a ФC31-derived integrative plasmid, which resulted in a recovered mycelial fragmentation at $18 \mathrm{~h}$ in mutant WYT-13, similar to that of the wild-type A. pretiosum ATCC 31280 (Figure 2D). 

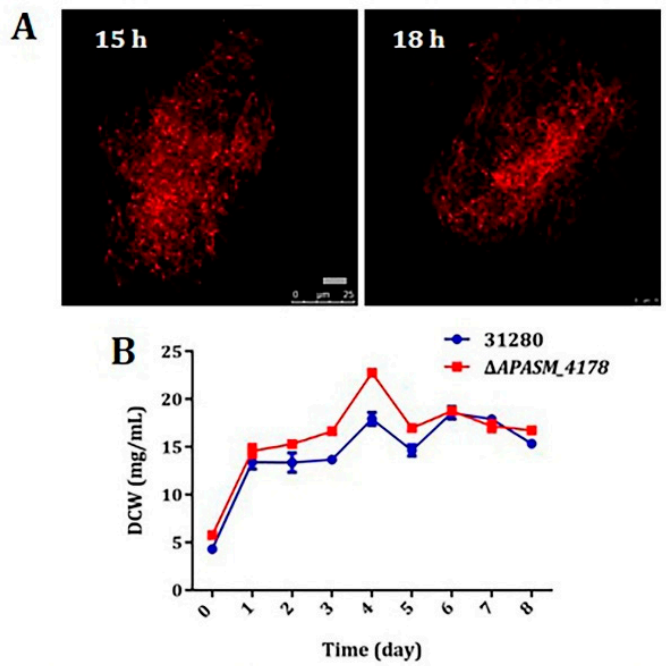

D
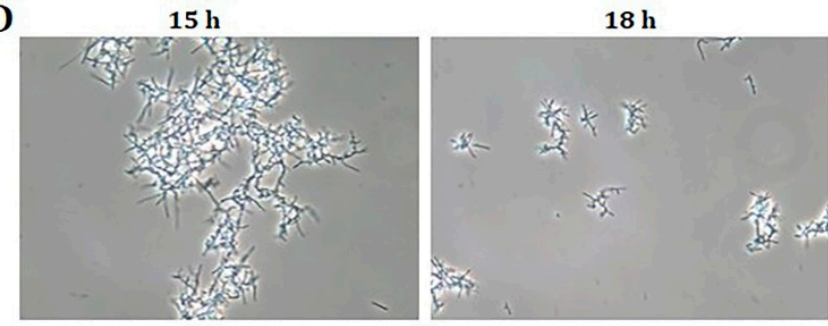

$48 \mathrm{~h}$

C ${ }^{80}$

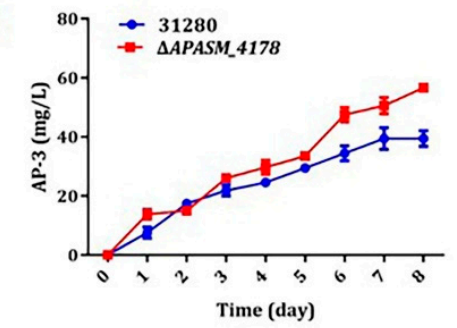

$24 \mathrm{~h}$

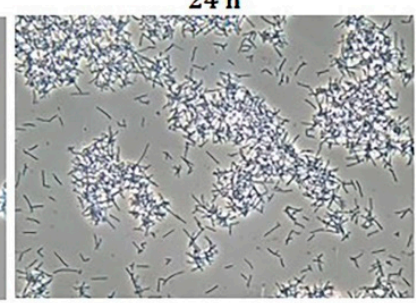

Figure 2. Deletion of subtilisin-like gene $A P A S M \_4178$ led to delayed mycelial fragmentation, increased growth and AP-3 yield. (A) Confocal micrographs of the APASM_4178-deleted mutant WYT-5. Scale bar: $10 \mu \mathrm{m}$. Magnification, 2500×; (B) Growth comparison between the wild-type and the mutant WYT-5. Error bars: mean \pm SD ( $n=3$ biological replicates); (C) AP-3 yield comparison between the wild-type and the mutant WYT-5. Error bars: mean \pm SD ( $n=3$ biological replicates); (D) Phase-contrast micrographs of WYT-5 complemented with cloned APASM_4178. Scale: $5 \mu \mathrm{m}$. Magnification, 400×.

\subsection{The Transcription of APASM_4178 and Mycelial Fragmentation is AdpA-Like-Dependent}

The recurrent fragmentation of $A$. pretiosum ATCC 31280 in early growth phase suggests an involvement of quorum sensing-like regulation (Figure 1B). The $\gamma$-butyrolactone system is the primary quorum sensing system in actinomycetes, and the widespread A-factor-dependent proteins (AdpA) mediate the regulatory signals of various $\gamma$-butyrolactone molecules and exert pleiotropic regulation on morphology differentiation and secondary metabolism $[44,45]$. Therefore, the upstream region of APASM_4178 was analyzed, and two conserved AdpA-binding motifs, $5^{\prime}$-TGACGGGGAG- ${ }^{\prime}$ and 5'-CGCGCCGCCA-3' (in reversed orientation), were identified (Figure 3A) [46].

Moreover, four genes, namely APASM_1021,APASM_3332,APASM_4306, and APASM_5462, were found to be annotated as AdpA-like-coding genes in the genome of $A$. pretiosum ATCC 31280 . However, they share a relatively low sequence identity between 39.62 and $51.46 \%$ (Supplementary Figure S4A). Subsequently all four genes were individually deleted through homologous recombination in $A$. pretiosum ATCC 31280, and the transcription of APASM_4178 in each mutant was analyzed through qRT-PCR. In the APASM_1021 deletion mutant WYT-15, the transcription of APASM_4178 was substantially reduced, whereas its transcription in the mutants of APASM_3332,APASM_4306, and APASM_5462 remained as high as that of the wild-type during the first 48-h cultivation (Figure 3B) (Supplementary Figure S4B). Accordingly, the mycelial morphology was examined under phase-contrast light microscope. As expected, the mycelial fragmentation occurred at $18 \mathrm{~h}$ in the wild-type but not in mutant WYT-15 (Figure 3C), suggesting that APASM_1021 is involved in the expression of APASM_4178. However, the production of AP-3 is abolished (data not shown) in WYT-15, which could be explained by the pleiotropic regulatory roles of AdpA-like proteins in actinomycetes [47]. 

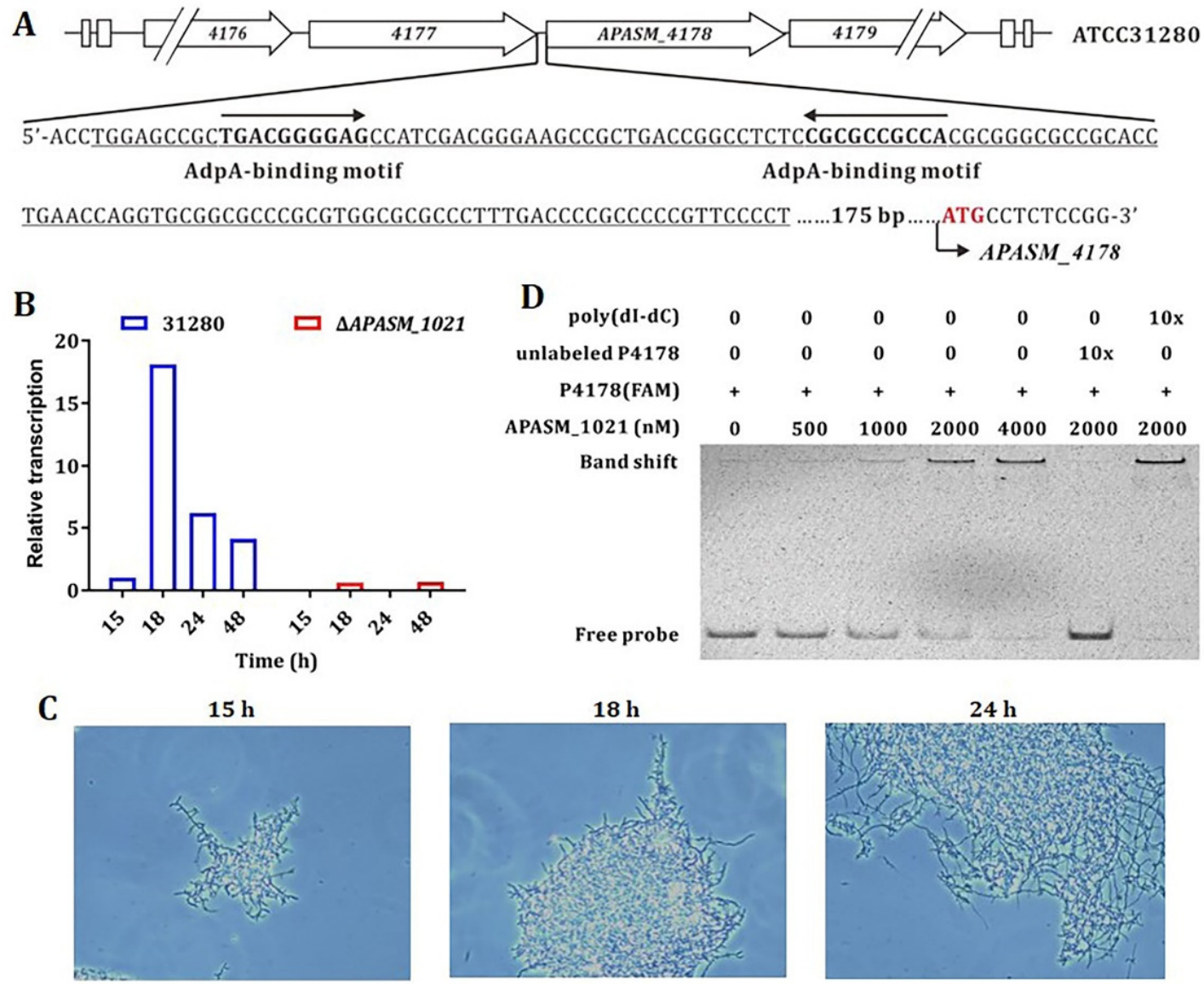

Figure 3. The transcription of APASM_4178 is regulated by an AdpA-like protein APASM_1021. (A) Conserved AdpA-binding motifs in the upstream region of APASM_4178. Underlined sequences refer to the 131-bp region covered by $\mathrm{P} 4178$, the $5^{\prime}$-FAM-labeled probe for electrophoretic mobility shift assay (EMSA). The start codon of APASM_4178 is shown in red; (B) The transcription of APASM_4178 in the wild-type and the APASM_1021-deleted mutant WYT-15. The transcription level of APASM_4178 in the wild-type at $15 \mathrm{~h}$ is set as 1 ; (C) Phase-contrast micrographs of the APASM_1021-deleted mutant WYT-15. Scale: $5 \mu \mathrm{m}$. Magnification, 400×. (D) EMSA of the specific binding between the upstream region of APASM_4178 and purified APASM_1021. P4178 is a 131-bp 5'-FAM-labeled probe.

Furthermore, an electrophoretic mobility shift assay (EMSA) was performed with purified His-tagged APASM_1021 and a 131-bp 5'-FAM-labelled DNA probe P4178, containing the two conserved AdpA-binding motifs within the putative promoter region of APASM_4178 (Figure 3A). APASM_1021 was found to bind the labeled probe P4178 at concentrations above $0.5 \mu \mathrm{M}$. When 10-fold of unlabeled probe P4178 was added to the binding mixture, the binding was competitively reversed, whereas a supplementation of 10-fold of nonspecific poly(dI-dC) had no effect (Figure 3D). The above-described results confirmed a specific regulation of the AdpA-like protein APASM_1021 on the expression of the subtilisin-like peptidase APASM_4178.

\subsection{Overexpression of Subtilisin Inhibitors Alleviated Mycelial Fragmentation and Increased AP-3 Production}

Subtilisin-like serine peptidases are widely spreaded in actinomycetes, and it is believed to be involved in morphology differentiation and other protein maturation processes. The activities of subtilisins are usually modulated by cognate subtilisin inhibitors (SSI) through specific binding [48,49]. Therefore, the genome sequence of $A$. pretiosum ATCC 31280 was analyzed and two SSI genes, APASM_3064 and APASM_6209, were identified. Since the presence of subtilisin-like APASM_4178 causes the mycelial fragmentation, these two SSI genes were individually overexpressed in A. pretiosum ATCC 31280 to test if there is an inhibition on the activity of APASM_4178 and consequent alleviation 
of mycelial fragmentation. As expected, the overexpression of each gene under the control of a strong $\mathrm{kasO}^{*}$ promoter led to a disappearance of mycelial fragmentation during the first 48-h cultivation (Figure 4A,B). Moreover, the overexpression of APASM_3064 led to $34.54 \%$ and $47.28 \%$ increase of biomass at day 5 and AP-3 yield, respectively, while the overexpression of APASM_6209 led to 42.60\% and $45.71 \%$ increase of biomass at day 5 and AP-3 yield, respectively, compared with those of the wild-type integrated with vector pBLQ856 (Figure 4C,D). However, the integration of $\Phi C 31$-derived plasmids caused decreased AP-3 yield for unknown reason.

A

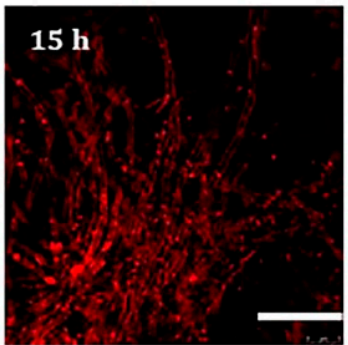

B

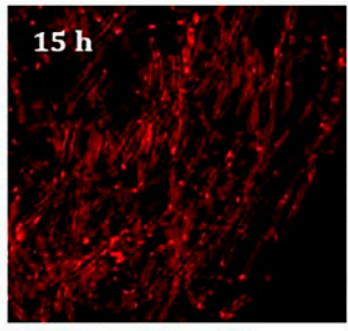

C
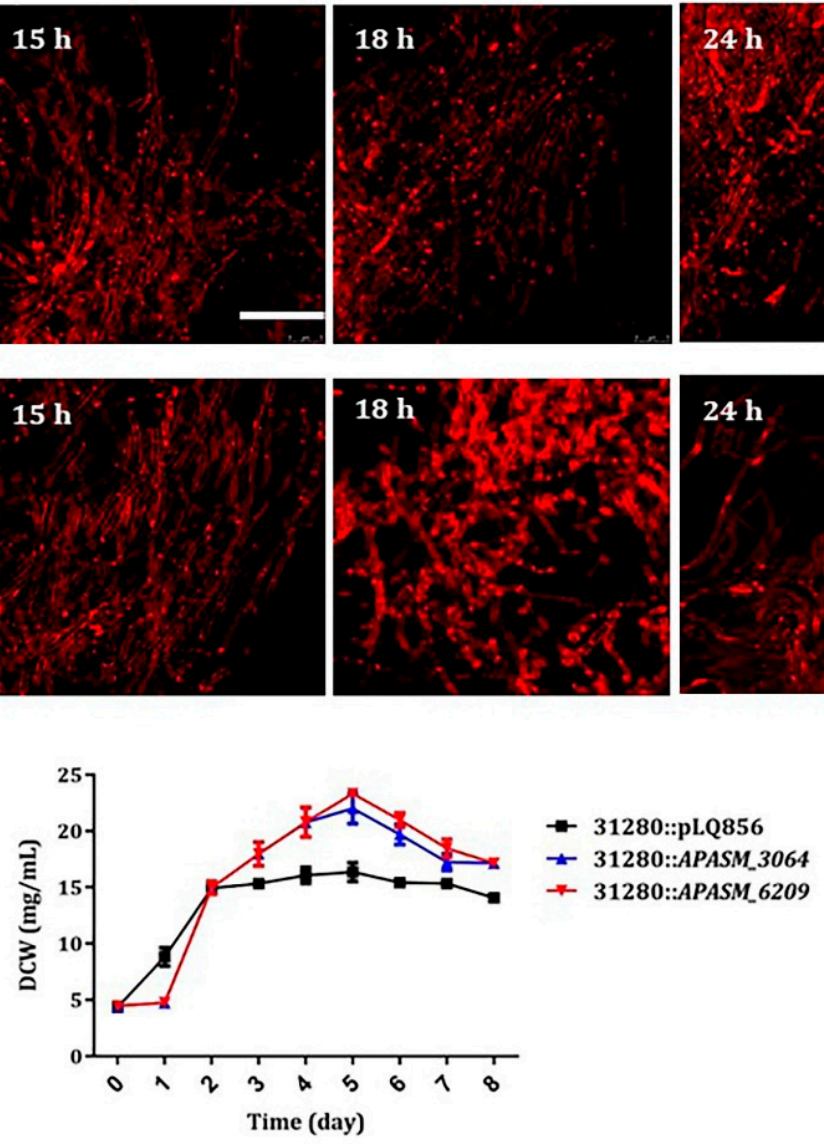

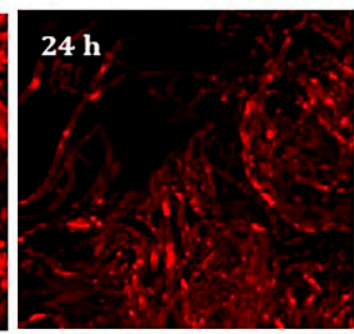

D

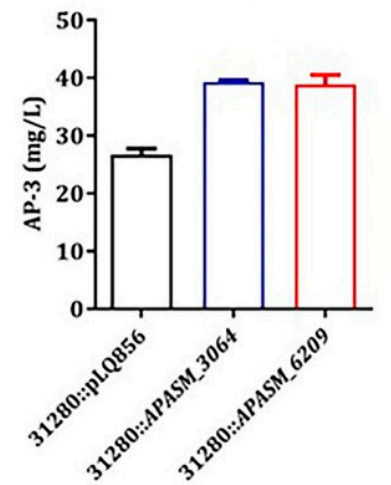

Figure 4. Overexpression of subtilisin inhibitor (SSI) genes in A. pretiosum ATCC 31280 alleviated mycelial fragmentation. (A) Confocal micrographs of mutant with SSI gene APASM_3064 overexpressed. Scale bar: $10 \mu \mathrm{m}$. Magnification, 2500×; (B) Confocal micrographs of mutant with SSI gene APASM_6209 overexpressed. Scale bar: $10 \mu \mathrm{m}$. Magnification, 2500×; (C) Growth comparison between 31280::pLQ856, the wild-type integrated with vector plasmid pLQ856, and mutants with APASM_3064 or APASM_3064 overexpressed. Error bars: mean $\pm \mathrm{SD}$ ( $n=3$ biological replicates); (D) Yield comparison between the control strain 31280::pLQ856 and mutants with APASM_3064 or APASM_6209 overexpressed. Error bars: mean $\pm \mathrm{SD}$ ( $n=3$ biological replicates).

3.5. Overexpression of APASM_4178 Led to Dispersed Mycelia and Improved Antibiotic Yields in Streptomyces Strains

Whereas A. pretiosum ATCC 31280 undergoes mycelial fragmentation at early stage of cultivation and has less biomass accumulation, most Streptomyces strains have robust growth and form large dense pellets consisting of interconnected hyphae, which usually limit the transfer of nutrients and oxygen and consequent antibiotic production [12]. In an attempt to reduce to the size of pellets, the subtilisin-like gene $A P A S M \_4178$ under the control of promoter $\mathrm{kasO}^{*}$ was introduced into salinomycin-producing Streptomyces albus BK 3-25 [50] and validamycin-producing Streptomyces hygroscopicus TL01 [51]. 
Interestingly, the overexpression of APASM_4178 in both strains indeed led to dispersed mycelia (Figure 5A,B). Consequently, the yield of salinomycin was increased by $33.80 \%$, from 17.99 to $24.07 \mathrm{~g} / \mathrm{L}$, and that of validamycin was increased by $14.94 \%$, from 18.67 to $21.46 \mathrm{~g} / \mathrm{L}$ (Figure 5C,D).

A

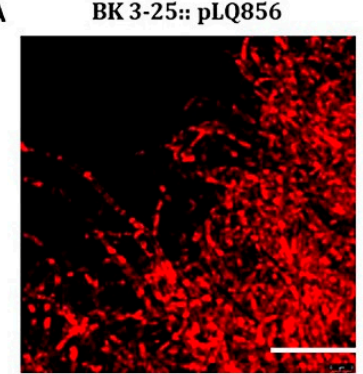

C
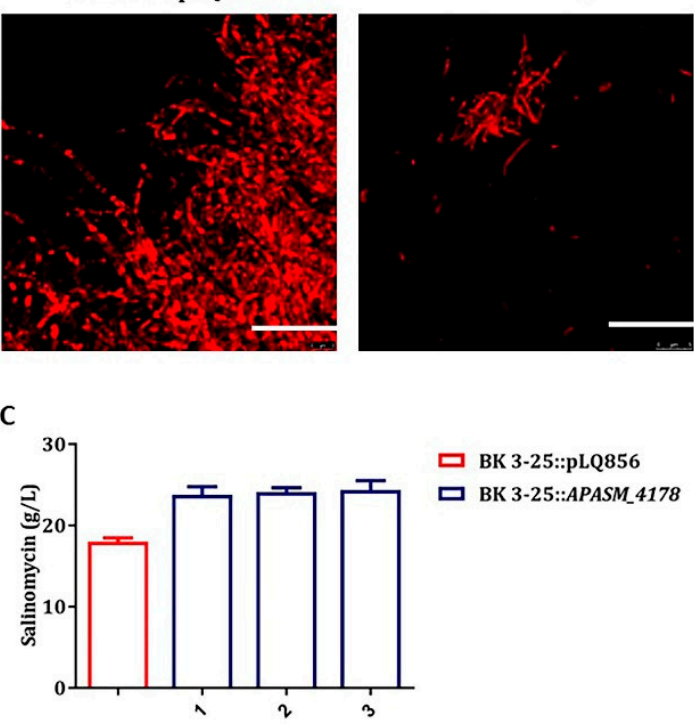

B

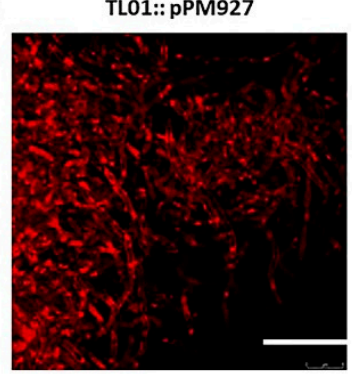

D

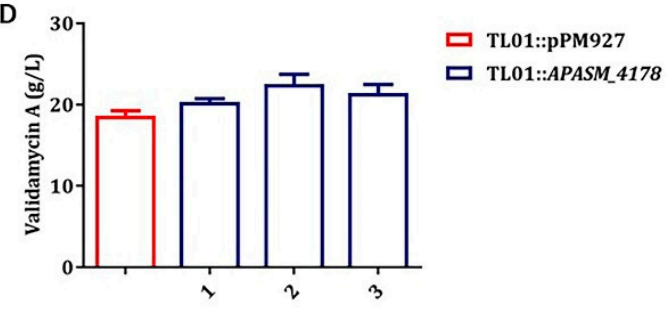

Figure 5. Overexpression of the subtilisin-like serine peptidase gene APASM_4178 in salinomycin-producing S. albus BK 3-25 and validamycin-producing S. hygroscopicus TL01. (A) Effect of APASM_4178 overexpression on the mycelial morphology of S. albus BK 3-25. Scale bars: $10 \mu \mathrm{m}$. Magnification, 2500×; (B) Effect of APASM_4178 overexpression on the mycelial morphology of S. hygroscopicus TL01. Scale bars: $10 \mu \mathrm{m}$. Magnification, 2500×; (C) Effect of APASM_4178 overexpression on salinomycin yield of $S$. albus BK 3-25. pLQ856: vector plasmid. Three mutant strains were randomly selected. Error bars: mean \pm SD ( $n=3$ biological replicates); (D) Effect of APASM_4178 overexpression on validamycin yield of $S$. hygroscopicus TL01. pPM927, vector plasmid. Three mutant strains were randomly selected. Error bars: mean $\pm \mathrm{SD}$ ( $n=3$ biological replicates).

\section{Discussion}

The mycelial fragmentation of A. pretiosum in liquid cultures has long been noticed [52] and even utilized for efficient selection of mutants through double crossover recombination [31]. However, when yield improvement was considered for future large-scale fermentation, the accompanying low biomass $(14.95 \mathrm{mg} / \mathrm{mL}$ ) with the mycelial fragmentation became a serious limit (Figure $1 \mathrm{~A})$. In order to eventually alleviate the fragmentation, RNA-seq transcriptome analysis was performed with samples collected with 3 or 6-h intervals, and the involved gene APASM_4178 was identified among 12 genes with the lowest transcription at $18 \mathrm{~h}$ of cultivation, the very moment of excessive fragmentation (Supplementary Table S3). Indeed, the deletion of APASM_4178 led to an alleviated fragmentation, $26.99 \%$ improved biomass and $43.65 \%$ increased AP-3 yield (Figure 2A-C). Our work displays the accuracy and efficiency of comparative transcriptome analysis in the identification of targets for genetic engineering, as also shown in the titer improvements of actinorhodin in S. coelicolor and of spiramycin in Streptomyces ambofaciens [53,54].

Gene APASM_4178 is 3,276 bp and predicted to code for a 1091-aa 120.0-kDa multi-domain protein, composed of a 150-aa N-terminal pro-peptide region, a 236-aa subtilisin-like serine peptidase domain, and a 616-aa C-terminal region. A BLAST search using APASM_4178 as a query identified more than 100 homologs with sequence identities higher than $40 \%$ and sequence coverage higher than 91\%. Moreover, most of them are from rare actinomycetes, including Actinosynnema, Saccharothrix, Lentzea, Saccharomonospora, Actinophytocola, and Micromonospora. Subtilisins are extracellular alkaline serine peptidases with a catalytic triad, consisting of Asp, His, and Ser, and are found to be widely 
involved in morphology differentiation and other protein maturation processes [48,49]. Different from APASM_4178 and its homologs, typical subtilisins from actinomycetes are smaller than $65.0 \mathrm{kDa}$ and contain an N-terminal pro-peptide and the serine peptidase domain [55]. Due to the presence of an extra 616-aa C-terminal region in APASM_4178, its proteolytic activity was measured and compared with APASM_6525, a 519-aa subtilisin-like serine peptidase from A. pretiosum ATCC 31280, and SCO1355, a 537-aa subtilisin-like serine peptidase from S. coelicolor A3(2) [49]. The results showed that APASM_4178 has similar peptidase activity to those of APASM_6525 and SCO1355, indicating that it is indeed a subtilisin-like serine peptidase (Supplementary Figure S5). However, the function of the large C-terminal region is still unknown and needs further investigation. Interestingly, the large C-terminal region of a cell envelope-located serine proteinase from Lactococcus lactis SK11 was proposed to be involved in anchoring of the enzyme in the cell membrane [56].

In actinomycetes, the expression of subtilisin and its cognate SSI is usually under the control of AdpA, the pleiotropic regulatory protein in the A-factor signaling cascade [57]. Even though APASM_4178 is substantially larger than typical subtilisins, its expression is similarly regulated by AdpA-like protein, as proved by in vivo deletion of AdpA-like coding gene and in vitro EMSA (Figure 3). The early and periodic occurrence of mycelial fragmentation in A. pretiosum ATCC 31280 actually resembles quorum sensing behavior responding to A-factor-like signal molecules. However, searching for A-factor and butanolide biosynthetic genes in the genome of A. pretiosum ATCC 31280, using scbA from S. coelicolor and sabA from Streptomyces ansochromogenes as queries, failed to retrieve any homologous genes, suggesting the presence of structurally different $\gamma$-butyrolactone in A. pretiosum ATCC 31280. Moreover, the alleviated mycelial fragmentation still happens after $48 \mathrm{~h}$ in the APASM_4178 deletion mutant WYT-5. When SSI genes were overexpressed in A. pretiosum ATCC 31280, the mycelial fragmentation did not occur, indicating the involvement of other subtilisin-like serine peptidase(s) in this process, which needs further investigation in the future.

In previous works for morphology engineering of actinomycetes, the cellulose synthase-like gene $\operatorname{csl} A$ [16], galactose oxidase-like gene glxA [17], PNAG synthase genes mat $A$ and matB [20], and amine oxidase gene hyaS [19] were deleted on one hand, and the cell-wall remodeling protein gene $\operatorname{ssg} A$ was overexpressed on the other hand. In our work, the large subtilisin-like serine peptidase gene APASM_4178 was used for the first time to engineer Streptomyces spp. for dispersed mycelia and improved antibiotic yields, which could be applied with many of other antibiotic-producing actinomycetes, which form dense pellets in submerged culture. In addition, the overexpression of SSI in A. pretiosum ATCC 31280 resulted in an improved AP-3 yield higher than that of the APASM_4178 deletion mutant. This strategy is applicable to some rare actinomycetes with mycelial fragmentation, especially those with homologous genes of APASM_4178.

\section{Conclusions}

This study identified a novel subtilisin-like serine peptidase gene by comparative transcriptome analysis in A. pretiosum ATCC 31280, and the deletion of this gene substantially reduced the mycelial fragmentation, increased biomass accumulation, and improved AP-3 yield. As a subtilisin-encoding gene, its transcription was controlled by a specific AdpA-like protein. Through the overexpression of subtilisin-binding SSI, the mycelial fragmentation was completely eliminated. The improved yields of AP-3, validamycin, and salinomycin along with altered morphologies of the producing strains highlighted new strategies for morphology engineering in actinomycetes.

Supplementary Materials: The following are available online at http://www.mdpi.com/2218-273X/10/6/851/s1, Table S1: Strains and plasmids used in this study; Table S2: Primers used in this study; Table S3: Compositions of five media used for morphology observation; Table S4: Candidate genes involved in mycelial fragmentation according to RNA-seq analysis; Figure S1: qRT-PCR verification of the transcription of genes selected by RNA-seq analysis; Figure S2: Deletion of gene APASM_4178; Figure S3: Mycelial morphology of mutants of 11 genes selected by RNA-seq analysis; Figure S4: Sequence alignments of four AdpA-like proteins and effects of their deletions on the transcription of APASM_4178; Figure S5: Protease activities of APASM_4178, APASM_6525, and SCO1355. 
Author Contributions: Conceptualization, L.B.; methodology, Y.W.; validation, Y.W; formal analysis, L.B. and Q.K.; investigation, Y.W.; data curation, L.B.; writing-original draft preparation, Y.W.; writing-review and editing, L.B.; supervision, L.B., Q.K. and L.-L.Z.; project administration, L.B.; funding acquisition, L.B. and L.-L.Z. All authors have read and agreed to the final version of the manuscript.

Funding: This research was funded by National Key R\&D Program of China (2019YFA0905400) and National Natural Science Foundation of China (U1703236, 31830104, 21661140002).

Acknowledgments: We are grateful to Xinjuan Ning and Xinran Wang for project discussion and Ping Zhang for the help on the laser confocal microscopy. We thank Tao Dong for revising the manuscript.

Conflicts of Interest: The authors declare no conflict of interest. The funders had no role in the design of the study; in the collection, analyses, or interpretation of data; in the writing of the manuscript, or in the decision to publish the results.

\section{References}

1. Flärdh, K.; Buttner, M.J. Streptomyces morphogenetics: Dissecting differentiation in a filamentous bacterium. Nat. Rev. Microbiol. 2009, 7, 36-49. [CrossRef] [PubMed]

2. Manteca, A.; Alvarez, R.; Salazar, N.; Yagüe, P.; Sanchez, J. Mycelium differentiation and antibiotic production in submerged cultures of Streptomyces coelicolor. Appl. Environ. Microbiol. 2008, 74, 3877-3886. [CrossRef] [PubMed]

3. Olmos, E.; Mehmood, N.; Haj Husein, L.; Goergen, J.L.; Fick, M.; Delaunay, S. Effects of bioreactor hydrodynamics on the physiology of Streptomyces. Bioprocess. Biosyst. Eng. 2013, 36, 259-272. [CrossRef] [PubMed]

4. Vecht-Lifshitz, S.E.; Sasson, Y.; Braun, S. Nikkomycin production in pellets of Streptomyces tendae. J. Appl. Bacteriol. 1992, 72, 195-200. [CrossRef]

5. Wardell, J.N.; Stocks, S.M.; Thomas, C.R.; Bushell, M.E. Decreasing the hyphal branching rate of Saccharopolyspora erythraea NRRL 2338 leads to increased resistance to breakage and increased antibiotic production. Biotechnol. Bioeng. 2002, 78, 141-146. [CrossRef]

6. Park, Y.; Tamura, S.; Koike, Y.; Toriyama, M.; Okabe, M. Mycelial pellet intrastructure visualization and viability prediction in a culture of Streptomyces fradiae using confocal scanning laser microscopy. J. Ferment. Bioeng. 1997, 84, 483-486. [CrossRef]

7. Jonsbu, E.; McIntyre, M.; Nielsen, J. The influence of carbon sources and morphology on nystatin production by Streptomyces noursei. J. Biotechnol. 2002, 95, 133-144. [CrossRef]

8. Kumar, P.; Dubey, K.K. Mycelium transformation of Streptomyces toxytricini into pellet: Role of culture conditions and kinetics. Bioresour. Technol. 2017, 228, 339-347. [CrossRef]

9. Ren, X.-D.; Chen, X.-S.; Tang, L.; Zeng, X.; Wang, L.; Mao, Z.-G. Physiological mechanism of the overproduction of $\varepsilon$-poly-L-lysine by acidic pH shock in fed-batch fermentation. Bioprocess. Biosyst. Eng. 2015, 38, 2085-2094. [CrossRef]

10. Yen, H.-W.; Hsiao, H.-P. Effects of dissolved oxygen level on rapamycin production by pellet-form of Streptomyces hygroscopicus. J. Biosci. Bioeng. 2013, 116, 366-370. [CrossRef]

11. van Veluw, G.J.; Petrus, M.L.; Gubbens, J.; de Graaf, R.; de Jong, I.P.; van Wezel, G.P.; Wosten, H.A.; Claessen, D. Analysis of two distinct mycelial populations in liquid-grown Streptomyces cultures using a flow cytometry-based proteomics approach. Appl. Microbiol. Biotechnol. 2012, 96, 1301-1312. [CrossRef] [PubMed]

12. van Dissel, D.; Claessen, D.; van Wezel, G.P. Chapter One-Morphogenesis of Streptomyces in submerged cultures. Adv. Appl. Microbiol. 2014, 89, 1-45. [PubMed]

13. Flärdh, K. Essential role of DivIVA in polar growth and morphogenesis in Streptomyces coelicolor A3(2). Mol. Microbiol. 2003, 49, 1523-1536. [CrossRef] [PubMed]

14. Holmes, N.A.; Walshaw, J.; Leggett, R.M.; Thibessard, A.; Dalton, K.A.; Gillespie, M.D.; Hemmings, A.M.; Gust, B.; Kelemen, G.H. Coiled-coil protein Scy is a key component of a multiprotein assembly controlling polarized growth in Streptomyces. Proc. Natl. Acad. Sci. USA 2013, 110, E397-E406. [CrossRef]

15. Kawamoto, S.; Watanabe, H.; Hesketh, A.; Ensign, J.C.; Ochi, K. Expression analysis of the $s s g A$ gene product, associated with sporulation and cell division in Streptomyces griseus. Microbiology 1997, 143, 1077-1086. [CrossRef]

16. Xu, H.; Chater, K.F.; Deng, Z.; Tao, M. A cellulose synthase-like protein involved in hyphal tip growth and morphological differentiation in Streptomyces. J. Bacteriol. 2008, 190, 4971-4978. [CrossRef] 
17. Liman, R.; Facey, P.D.; van Keulen, G.; Dyson, P.J.; Del Sol, R. A laterally acquired galactose oxidase-like gene is required for aerial development during osmotic stress in Streptomyces coelicolor. PLoS ONE 2013, 8, e54112. [CrossRef]

18. Kwak, J.; McCue, L.A.; Trczianka, K.; Kendrick, K.E. Identification and characterization of a developmentally regulated protein, EshA, required for sporogenic hyphal branches in Streptomyces griseus. J. Bacteriol. 2001, 183, 3004-3015. [CrossRef]

19. Koebsch, I.; Overbeck, J.; Piepmeyer, S.; Meschke, H.; Schrempf, H. A molecular key for building hyphae aggregates: The role of the newly identified Streptomyces protein HyaS. Microb. Biotechnol. 2009, 2, 343-360. [CrossRef]

20. van Dissel, D.; Willemse, J.; Zacchetti, B.; Claessen, D.; Pier, G.B.; van Wezel, G.P. Production of poly- $\beta-1,6-N$-acetylglucosamine by MatAB is required for hyphal aggregation and hydrophilic surface adhesion by Streptomyces. Microb Cell 2018, 5, 269-279. [CrossRef]

21. Nguyen, H.T.; Pham, V.T.T.; Nguyen, C.T.; Pokhrel, A.R.; Kim, T.-S.; Kim, D.; Na, K.; Yamaguchi, T.; Sohng, J.K. Exploration of cryptic organic photosensitive compound as Zincphyrin IV in Streptomyces venezuelae ATCC 15439. Appl. Microbiol. Biotechnol. 2020, 104, 713-724. [CrossRef] [PubMed]

22. van Wezel, G.P.; Krabben, P.; Traag, B.A.; Keijser, B.J.F.; Kerste, R.; Vijgenboom, E.; Heijnen, J.J.; Kraal, B. Unlocking Streptomyces spp. for use as sustainable industrial production platforms by morphological engineering. Appl. Environ. Microbiol. 2006, 72, 5283-5288. [CrossRef] [PubMed]

23. Kupchan, S.M.; Komoda, Y.; Court, W.A.; Thomas, G.J.; Smith, R.M.; Karim, A.; Gilmore, C.J.; Haltiwanger, R.C.; Bryan, R.F. Maytansine, a novel antileukemic ansa macrolide from Maytenus ovatus. J. Am. Chem. Soc. 1972, 94, 1354-1356. [CrossRef] [PubMed]

24. Higashide, E.; Asai, M.; Ootsu, K.; Tanida, S.; Kozai, Y.; Hasegawa, T.; Kishi, T.; Sugino, Y.; Yoneda, M. Ansamitocin, a group of novel maytansinoid antibiotics with antitumour properties from Nocardia. Nature 1977, 270, 721-722. [CrossRef] [PubMed]

25. Land, M.; Lapidus, A.; Mayilraj, S.; Chen, F.; Copeland, A.; Del Rio, T.G.; Nolan, M.; Lucas, S.; Tice, H.; Cheng, J.-F.; et al. Complete genome sequence of Actinosynnema mirum type strain (101T). Stand. Genom. Sci. 2009, 1, 46-53. [CrossRef] [PubMed]

26. Snipes, C.E.; Duebelbeis, D.O.; Olson, M.; Hahn, D.R.; Dent, W.H.; Gilbert, J.R.; Werk, T.L.; Davis, G.E.; Lee-Lu, R.; Graupner, P.R. The ansacarbamitocins: Polar ansamitocin derivatives. J. Nat. Prod. 2007, 70, 1578-1581. [CrossRef] [PubMed]

27. Li, X.-M.; Li, X.-M.; Lu, C.-H. Abscisic acid-type sesquiterpenes and ansamycins from Amycolatopsis alba DSM 44262. J. Asian Nat. Prod. Res. 2017, 19, 946-953. [CrossRef] [PubMed]

28. Zhang, Y.G.; Liu, Q.; Wang, H.F.; Park, D.J.; Guo, J.W.; Kim, C.J.; Zhang, Y.M.; Li, W.J. Nocardiopsis ansamitocini sp. nov., a new producer of ansamitocin P-3 of the genus Nocardiopsis. Int. J. Syst. Evol. Microbiol. 2016, 66, 230-235. [CrossRef]

29. Mecklenburg, L. A brief introduction to antibody-drug conjugates for toxicologic pathologists. Toxicol. Pathol. 2018, 46, 746-752. [CrossRef]

30. Lambert, J.M.; Chari, R.V.J. Ado-trastuzumab Emtansine (T-DM1): An antibody-drug conjugate (ADC) for HER2-positive breast cancer. J. Med. Chem. 2014, 57, 6949-6964. [CrossRef]

31. Yu, T.-W.; Bai, L.; Clade, D.; Hoffmann, D.; Toelzer, S.; Trinh, K.Q.; Xu, J.; Moss, S.J.; Leistner, E.; Floss, H.G. The biosynthetic gene cluster of the maytansinoid antitumor agent ansamitocin from Actinosynnema pretiosum. Proc. Natl. Acad. Sci. USA 2002, 99, 7968-7973. [CrossRef] [PubMed]

32. Kang, Q.; Shen, Y.; Bai, L. Biosynthesis of 3,5-AHBA-derived natural products. Nat. Prod. Rep. 2012, 29, 243-263. [CrossRef] [PubMed]

33. Li, T.; Fan, Y.; Nambou, K.; Hu, F.; Imanaka, T.; Wei, L.; Hua, Q. Improvement of ansamitocin P-3 production by Actinosynnema mirum with fructose as the sole carbon source. Appl. Biochem. Biotechnol. 2015, 175, 2845-2856. [CrossRef] [PubMed]

34. Fan, Y.; Gao, Y.; Zhou, J.; Wei, L.; Chen, J.; Hua, Q. Process optimization with alternative carbon sources and modulation of secondary metabolism for enhanced ansamitocin P-3 production in Actinosynnema pretiosum. J. Biotechnol. 2014, 192, 1-10. [CrossRef] [PubMed]

35. Bandi, S.; Kim, Y.; Chang, Y.K.; Shang, G.D.; Yu, T.W.; Floss, H.G. Construction of asm2 deletion mutant of Actinosynnema pretiosum and medium optimization for ansamitocin P-3 production using statistical approach. J. Microbiol. Biotechnol. 2006, 16, 1338-1346. 
36. Zhao, M.; Fan, Y.; Wei, L.; Hu, F.; Hua, Q. Effects of the methylmalonyl-CoA metabolic pathway on ansamitocin production in Actinosynnema pretiosum. Appl. Biochem. Biotechnol. 2017, 181, 1167-1178. [CrossRef]

37. Fan, Y.; Hu, F.; Wei, L.; Bai, L.; Hua, Q. Effects of modulation of pentose-phosphate pathway on biosynthesis of ansamitocins in Actinosynnema pretiosum. J. Biotechnol. 2016, 230, 3-10. [CrossRef]

38. Du, Z.-Q.; Zhong, J.-J. Rational approach to improve ansamitocin P-3 production by integrating pathway engineering and substrate feeding in Actinosynnema pretiosum. Biotechnol. Bioeng. 2018, 115, 2456-2466. [CrossRef]

39. Ning, X.; Wang, X.; Wu, Y.; Kang, Q.; Bai, L. Identification and engineering of post-PKS modification bottlenecks for ansamitocin P-3 titer improvement in Actinosynnema pretiosum subsp. pretiosum ATCC 31280. Biotechnol. J. 2017, 12, 1700484. [CrossRef]

40. Kieser, T.; Bibb, M.J.; Buttner, M.J.; Chater, K.F.; Hopwood, D.A. Practical Streptomyces Genetics; John Innes Foundation: Norwich, UK, 2000.

41. Livak, K.J.; Schmittgen, T.D. Analysis of relative gene expression data using real-time quantitative PCR and the $2^{-\Delta \Delta C T}$ Method. Methods 2001, 25, 402-408. [CrossRef]

42. Hellman, L.M.; Fried, M.G. Electrophoretic mobility shift assay (EMSA) for detecting protein-nucleic acid interactions. Nat. Protoc. 2007, 2, 1849-1861. [CrossRef] [PubMed]

43. Qu, S.; Kang, Q.; Wu, H.; Wang, L.; Bai, L. Positive and negative regulation of GlnR in validamycin A biosynthesis by binding to different loci in promoter region. Appl. Microbiol. Biotechnol. 2015, 99, 4771-4783. [CrossRef] [PubMed]

44. Vujaklija, D.; Horinouchi, S.; Beppu, T. Detection of an A-factor-responsive protein that binds to the upstream activation sequence of $s t r R$, a regulatory gene for streptomycin biosynthesis in Streptomyces griseus. J. Bacteriol. 1993, 175, 2652-2661. [CrossRef] [PubMed]

45. Rabyk, M.; Yushchuk, O.; Rokytskyy, I.; Anisimova, M.; Ostash, B. Genomic insights into evolution of AdpA family master regulators of morphological differentiation and secondary metabolism in Streptomyces. J. Mol. Evol. 2018, 86, 204-215. [CrossRef] [PubMed]

46. Yamazaki, H.; Tomono, A.; Ohnishi, Y.; Horinouchi, S. DNA-binding specificity of AdpA, a transcriptional activator in the A-factor regulatory cascade in Streptomyces griseus. Mol. Microbiol. 2004, 53, 555-572. [CrossRef] [PubMed]

47. Horinouchi, S.; Beppu, T. Hormonal control by A-factor of morphological development and secondary metabolism in Streptomyces. Proc. Jpn. Acad. Ser. B Phys. Biol. Sci. 2007, 83, 277-295. [CrossRef] [PubMed]

48. Satow, Y.; Mitsui, Y.; Iitaka, Y.; Murao, S.; Sato, S. Crystallization and preliminary X-ray investigation of a new alkaline protease inhibitor and its complex with subtilisin BPN'. J. Mol. Biol. 1973, 75, 745-IN22. [CrossRef]

49. Kim, D.W.; Hesketh, A.; Kim, E.S.; Song, J.Y.; Lee, D.H.; Kim, I.S.; Chater, K.F.; Lee, K.J. Complex extracellular interactions of proteases and a protease inhibitor influence multicellular development of Streptomyces coelicolor. Mol. Microbiol. 2008, 70, 1180-1193. [CrossRef]

50. Zhang, X.; Lu, C.; Bai, L. Mechanism of salinomycin overproduction in Streptomyces albus as revealed by comparative functional genomics. Appl. Microbiol. Biotechnol. 2017, 101, 4635-4644. [CrossRef]

51. Tan, G.-Y.; Bai, L.; Zhong, J.-J. Exogenous 1,4-butyrolactone stimulates A-factor-like cascade and validamycin biosynthesis in Streptomyces hygroscopicus 5008. Biotechnol. Bioeng. 2013, 110, 2984-2993. [CrossRef]

52. Watanabe, K.; Okuda, T.; Yokose, K.; Furumai, T.; Maruyama, H.B. Actinosynnema mirum, a new producer of nocardicin antibiotics. J. Antibiot. 1983, 36, 321-324. [CrossRef] [PubMed]

53. Kim, M.W.; Lee, B.R.; You, S.; Kim, E.J.; Kim, J.N.; Song, E.; Yang, Y.H.; Hwang, D.; Kim, B.G. Transcriptome analysis of wild-type and $a f_{s} S$ deletion mutant strains identifies synergistic transcriptional regulator of $a f_{s} S$ for a high antibiotic-producing strain of Streptomyces coelicolor A3(2). Appl. Microbiol. Biotechnol. 2018, 102, 3243-3253. [CrossRef] [PubMed]

54. Fondi, M.; Pinatel, E.; Talà, A.; Damiano, F.; Consolandi, C.; Mattorre, B.; Fico, D.; Testini, M.; De Benedetto, G.E.; Siculella, L.; et al. Time-resolved transcriptomics and constraint-based modeling identify system-level metabolic features and overexpression targets to increase spiramycin production in Streptomyces ambofaciens. Front. Microbiol. 2017, 8, 835. [CrossRef] [PubMed]

55. Suzuki, M.; Taguchi, S.; Yamada, S.; Kojima, S.; Miura, K.I.; Momose, H. A novel member of the subtilisin-like protease family from Streptomyces albogriseolus. J. Bacteriol. 1997, 179, 430-438. [CrossRef] [PubMed] 
56. Vos, P.; Simons, G.; Siezen, R.J.; de Vos, W.M. Primary structure and organization of the gene for a procaryotic, cell envelope-located serine proteinase. J. Biol. Chem. 1989, 264, 13579-13585. [PubMed]

57. Hirano, S.; Kato, J.Y.; Ohnishi, Y.; Horinouchi, S. Control of the Streptomyces subtilisin inhibitor gene by AdpA in the A-factor regulatory cascade in Streptomyces griseus. J. Bacteriol. 2006, 188, 6207-6216. [CrossRef]

(C) 2020 by the authors. Licensee MDPI, Basel, Switzerland. This article is an open access article distributed under the terms and conditions of the Creative Commons Attribution (CC BY) license (http://creativecommons.org/licenses/by/4.0/). 